\title{
A RELATIVISTIC MODEL OF THE GALACTIC ROTATION
}

\author{
Nota del m.e. ANGELO LOINGER (*) e TIZIANA MARSICO (**)
}

(Adunanza del 26 novembre 2015)

Sunto. - Si dimostra con un modello di relatività generale che la materia oscura può essere sostituita da una forza gravitazionale-rotazionale. Si è considerato particolareggiatamente il caso della nostra galassia, la Via Lattea.

Abstract. - We prove with a general-relativistic model that the Dark Matter can be substituted by a gravitational-rotational force. To be determinate, we have considered the instance of the Milky Way.

SummaRY. - Introduction. - 1. An observational diagram of the circular velocity curves of the Milky Way stars, and our theoretical counterpart. - 2. Mathematical development of our GR-model. - 3, Observational and theoretical data of the model. - 4. Conclusion.

PPACS:04.20 - General relativity; 9850 and 9860 - Galaxies; 98.50L - Milky Way.

INTRODUCTION. - Let us consider the ensemble of the stellar revolutions in a galaxy around its supermassive centre. As it is well known, the star trajectories are rather peculiar. In particular, the stars that are very far from the centre seem to obey Newton's law of gravitation only if one hypothesizes the existence of an invisible, ad hoc, Dark Matter Halo.

To be determinate, in the present Note we study the instance of a given spiral galaxy, our MILKY WAY. We prove, with a convenient relativistic approach, that the joined action of the galactic rotation and of gravity yields a behaviour of the far-star orbits which is in a good agreement with the observational data.

(*) Dipartimento di Fisica, Università di Milano, Via Celoria, 16 - 20133 Milano (Italy). Email angelo.loinger@mi.infn.it

(**) Liceo Classico “G. Berchet”, Via della Commenda, 26 - 20122 Milano (Italy). Email: martiz64@libero.it 


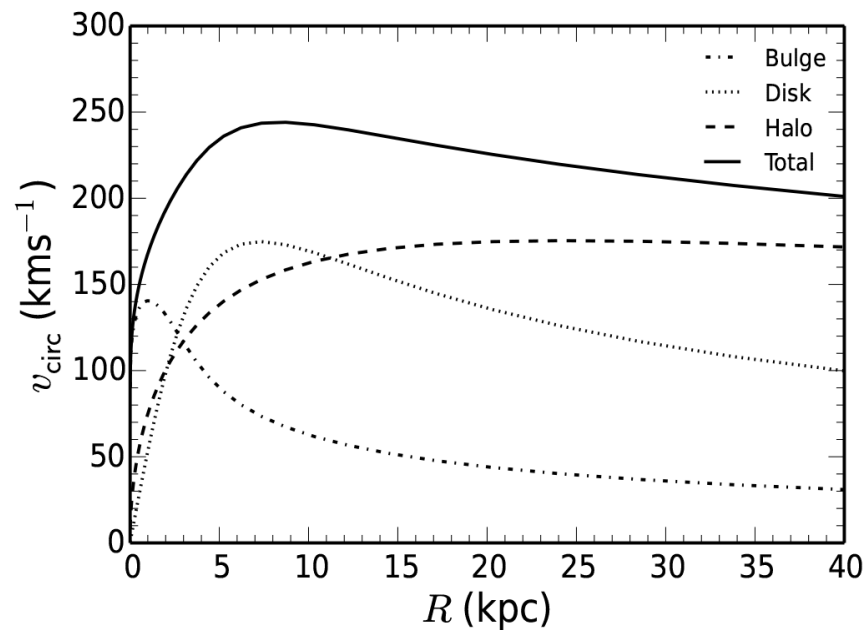

Fig. 1. The circular velocity curve of the Milky Way (Fig. 8 of paper [1]). The circular velocity curve of the Galaxy: the dotted, dashed-dotted and dashed lines are the circular velocity curves along the meridional plane, $z=0$, for the oblate bulge, Miyamoto-Nagai disk and NFW halo respectively. The radius $R$ is the distance in the Galactic plane. The individual curves are constructed from the best fit estimates of the model parameters. The solid line shows the resultant circular velocity curves due to all the three components of the Galaxy.

1. - In our Fig. 1, we have reproduced (with its legend) the Fig. 8 of paper [1]. We are interested in the solid-line diagram, which represents the circular velocity curves of all the Milky Way stars. We distinguish in it two parts. In the ensemble of the stars that are nearer to the galactic centre, from $\mathrm{SgrA}^{*}$ to the distance $R=8 \mathrm{kpc}$, the star velocities increase linearly with the distance from the centre (as in the rotation of a solid body). In the ensemble of the very far stars, from $R=8$ to $R=40 \mathrm{kpc}$, the velocities decrease slightly, and tend to become constant.

The following relativistic model explains these behaviours. However, the part of the theoretical diagram of Fig. 2 which regards the stars of the first ensemble is different from the analogous part of the observational diagram of Fig. 1, being similar in form to the first part of a diagram reproduced in the treatise by Unsöld and Baschek [2]. 


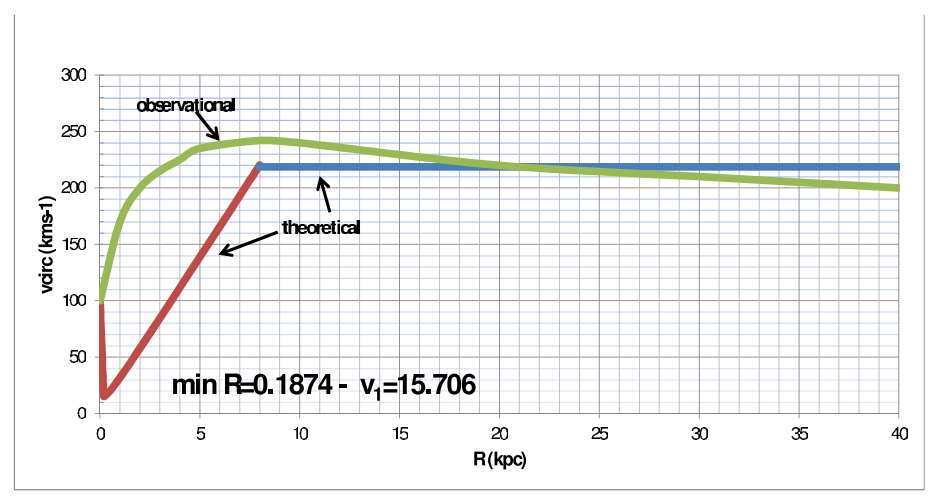

Fig. 2. The theoretical red line represents $v_{1}(\mathcal{R})$ of formula (6).The theoretical blue line represents $v_{2}(\mathcal{R})$ of formula ((14)).

2. - We start from the spacetime interval $\mathrm{d} s$ of the Schwarzschild manifold created by a concentrated mass $M$ :

(1) $\mathrm{d} s^{2}=\left(\frac{\mathcal{R}-\alpha}{\mathcal{R}}\right) c^{2} \mathrm{~d} t^{2}-\left(\frac{\mathcal{R}}{\mathcal{R}-\alpha}\right) \mathrm{d} \mathcal{R}^{2}-\mathcal{R}^{2}\left(\mathrm{~d} \vartheta^{2}+\sin ^{2} \vartheta \mathrm{d} \varphi^{2}\right)$, where $\mathcal{R}=\mathcal{R}(r)$ is any regular function of the radial coordinate $r,(0 \leq$ $r<\infty)$, which gives a Minkowskian manifold if $r \rightarrow \infty ; \alpha \equiv 2 m$; $m \equiv$ $G M / c^{2}$. We are interested in the circular $(\mathrm{d} \mathcal{R}=0)$ geodesics of the testparticles (which represent the stars in our model) and of the light-rays, moving in the plane $\vartheta=\pi / 2$.

If $\Omega(>0)$ is the partial common angular velocity of the stars of the first ensemble (from $\mathrm{SgrA}^{*}$ to the distance of $8 \mathrm{kpc}$ ), we put $\varphi \rightarrow \varphi-\Omega t$. Thus eq. (1) becomes:

$$
\left(\mathrm{d} s^{2}\right)_{1}=\left(\frac{\mathcal{R}-\alpha}{\mathcal{R}}-\mathcal{R}^{2} \frac{\Omega^{2}}{c^{2}}\right) c^{2} \mathrm{~d} t^{2}-\mathcal{R}^{2} \mathrm{~d} \varphi^{2}+2 \mathcal{R}^{2} \Omega \mathrm{d} \varphi \mathrm{d} t ;
$$

obviously,

$$
\frac{\mathcal{R}-\alpha}{\mathcal{R}^{3}}>\frac{\Omega^{2}}{c^{2}}
$$

Let us consider the following Lagrangian $\mathcal{L}_{1}$ :

(3) $\mathcal{L}_{1}=\frac{\left(\mathrm{d} s^{2}\right)_{1}}{c^{2} \mathrm{~d} t^{2}}=\frac{\mathcal{R}-\alpha}{\mathcal{R}}-\mathcal{R}^{2} \frac{\Omega^{2}}{c^{2}}-\frac{\mathcal{R}^{2}}{c^{2}} \dot{\varphi}^{2}+2 \mathcal{R}^{2} \frac{\Omega}{c^{2}} \dot{\varphi}=A_{1} \geq 0$, where $\dot{\varphi} \equiv \mathrm{d} \varphi / \mathrm{d} t$. 
We have:

$$
\begin{gathered}
\frac{\partial \mathcal{L}_{1}}{\partial \mathcal{R}}=0=\frac{\alpha}{\mathcal{R}^{2}}-2 \mathcal{R} \frac{\Omega^{2}}{c^{2}}-2 \frac{\mathcal{R}}{c^{2}} \dot{\varphi}^{2}+4 \frac{\mathcal{R} \Omega}{c^{2}} \dot{\varphi} ; \Rightarrow \\
\dot{\varphi}^{2}-2 \Omega \dot{\varphi}+\Omega^{2}-\frac{\alpha c^{2}}{2 \mathcal{R}^{3}}=0 ; \Rightarrow \\
\dot{\varphi}=\Omega \pm \frac{c}{\mathcal{R}} \sqrt{\frac{\alpha}{2 \mathcal{R}}} ;
\end{gathered}
$$

for evident reasons, we choose the positive sign; thus:

$$
v_{1}=(\mathcal{R} \dot{\varphi})_{1}=\mathcal{R} \Omega+c \sqrt{\frac{\alpha}{2 \mathcal{R}}} .
$$

If we substitute $\mathcal{R} \dot{\varphi}$ of eq. (6) in $\mathcal{L}_{1}$, we obtain a new positive inequality for the test-particles $\left(A_{1}>0\right)$; it follows (Hilbertian gravitational repulsion [3]):

$$
\mathcal{R}>\frac{3}{2} \alpha
$$

as in the instance $\Omega=0([3])$ - and further:

$$
(\mathcal{R} \dot{\varphi})_{1}-\mathcal{R} \Omega<\frac{c}{\sqrt{3}} .
$$

For the light-rays:

$$
\mathcal{R}=\frac{3}{2} \alpha ; \quad(\mathcal{R} \dot{\varphi})_{1}-\mathcal{R} \Omega=\frac{c}{\sqrt{3}} \cdot-
$$

For the stars of the second ensemble (from $\mathcal{R}=8$ to $\mathcal{R}=40$ ), we put ( $u$ is a positive constant):

$$
\varphi \rightarrow \varphi-\frac{u}{R} t ; \quad \Rightarrow \dot{\varphi} \rightarrow \dot{\varphi}-\frac{u}{R}
$$

because $\mathrm{d} \mathcal{R}=0$.

We have the following Lagrangian $\mathcal{L}_{2}$ :

(11) $\mathcal{L}_{2}=\frac{\left(\mathrm{d} s^{2}\right)_{2}}{c^{2} \mathrm{~d} t^{2}}=\frac{\mathcal{R}-\alpha}{\mathcal{R}}-\frac{\mathcal{R}^{2}}{c^{2}}\left(\dot{\varphi}^{2}+\frac{u^{2}}{\mathcal{R}^{2}}-2 \frac{u \dot{\varphi}}{\mathcal{R}}\right)=A_{2} \geq 0$.

The agreement at $\mathcal{R}=\tilde{\mathcal{R}}(=8 \mathrm{kpc})$ between $\left(\mathrm{d} s^{2}\right)_{1}$ and $\left(\mathrm{d} s^{2}\right)_{2}$ gives:

$$
u=\tilde{\mathcal{R}} \Omega \text {. }
$$


Then

$$
\begin{gathered}
\frac{\partial \mathcal{L}_{2}}{\partial \mathcal{R}}=0=\frac{\alpha}{\mathcal{R}^{2}}-2 \frac{\mathcal{R}}{c^{2}} \dot{\varphi}^{2}+2 \frac{\tilde{\mathcal{R}} \Omega}{c^{2}} \dot{\varphi} ; \quad \Rightarrow \\
v_{2} \equiv(\mathcal{R} \dot{\varphi})_{2}=\frac{\tilde{\mathcal{R}} \Omega}{2}+\sqrt{\frac{\tilde{\mathcal{R}}^{2} \Omega^{2}}{4}+\frac{\alpha c^{2}}{2 \mathcal{R}}},
\end{gathered}
$$

where we have chosen the positive sign before the root. -

The theoretical diagram of Fig. 2 represents formulae (6) (near stars) and (14) (far stars). It has been drawn by employing the radial coordinate $\mathcal{R}$ as a reasonable substitute (in this problem) of the distance $R$ - see Hilbert [3] and Appendix B in [4].

3. - We have employed the following numerical parameters:

$$
\begin{gathered}
M \equiv M_{\mathrm{SgrA}^{*}}=4.5 \times 10^{6} M_{\odot} ; \Rightarrow \\
\alpha=3 \mathrm{~km} \times 4.5 \times 10^{6}=1.35 \times \times 10^{7} \mathrm{~km} .
\end{gathered}
$$

Angular velocity $\Omega$ of the galaxy rotation:

$$
\Omega=2 \pi / T_{\odot},
$$

where $T_{\odot}$ is the period of the solar revolutions around $\mathrm{SgrA}^{*}$; Sun's velocity: $220 \mathrm{kms}^{-1}$. This period has been conventionally computed with the Newtonian law of attraction between $M_{\mathrm{SgrA}^{*}}$ and $M_{\odot}$. We have: $T_{\odot}=225 \times 10^{6} \mathrm{yr}$; thus:

$$
\Omega=8.855 \times 10^{-16} \mathrm{rads}^{-1} .
$$

We assume that this $\Omega$ gives a good approximation of the real angular velocity of the milky Way.

We remember finally that

$$
1 \mathrm{kpc} \hat{=} 3.087 \times 10^{16} \mathrm{~km} .-
$$

The function $v_{1}(\mathcal{R})$ of formula (6) has a minimum at the following value $\mathcal{R}_{\text {min }}$ of $\mathcal{R}$ :

$$
\begin{gathered}
\mathcal{R}_{\text {min }}=\frac{1}{2}\left(\frac{\alpha c^{2}}{\Omega^{2}}\right)^{1 / 3} ; \Rightarrow \\
v_{1}\left(\mathcal{R}_{\text {min }}\right)=\frac{1}{2}\left(\alpha c^{2} \Omega\right)^{1 / 3}+c \sqrt{\alpha}\left(\frac{\alpha c^{2}}{\Omega^{2}}\right)^{-1 / 6} ; \Rightarrow
\end{gathered}
$$




$$
\begin{gathered}
\dot{\varphi}_{1}\left(\mathcal{R}_{\mathrm{min}}\right)=3 \Omega . \\
v_{1}(\mathcal{R} \approx 1 \mathrm{pc})=100 \mathrm{kms}^{-1}:
\end{gathered}
$$

the common initial point of the two diagrams of Fig. 2.

4. - We think that our result shows the possibility that Einstein equations of general relativity contain solutions which can substitute the Dark Matter of the galaxies with an inertial-gravitational force. -

$$
\begin{array}{r}
\text { "Par l'espace, l'univers } \\
\text { me comprend et m'engloutit } \\
\text { comme un point; } \\
\text { par la pensée, je le comprends." } \\
\text { B. Pascal }
\end{array}
$$

\section{REFERENCES}

[1] P.R. Kafle et al., arXiv:1408.1787 [Astro-ph.GA] 8 August 2014.

[2] A. Unsöld and B. Baschek, The New Cosmos (Springer-Verlag, Berlin, etc.) 2002, p.412.

[3] D. Hilbert, Mathem. Annalen, 92 (1924) 1; also in Gesammelte Abhandlungen, Dritter Band (J. Springer, Berlin) 1935, p.258.

[4] A. Loinger and T. Marsico, arXiv:1011.2600 [physics.gen-ph] 11 Nov 2010. 